\title{
COMMENTARY ON IMPORTANCE OF CARDIAC ENZYME TESTING - BIOCHEMIST PERCEPTIVE
}

\author{
Rachel Jacob
}

The diagnosis of Acute Myocardial Infarction by WHO requires at least two of the following evidences: 1 . History of chest pain, 2.Evolutionary changes in ECG, 3. Elevation of serial cardiac enzymes and proteins; referred to as cardiac biomarkers.

The damaged myocardial tissue in Acute MI, release enzymes CK-MB, LDH, AST into the blood stream during the window period, while the proteins: cardiac Troponins, myoglobin are released from the necrotic myocardium. These elevations are succeeded by the evolving ECG changes. The joint European Society of Cardiologists and the American College of Cardiologists in 2000 modified the definition of acute MI and now requires a rise and fall in biochemical markers together with ischemic symptoms such as development pathological $Q$ waves, ischemic ECG changes which has thus shifted the onus to biochemical evidence. Hence the contribution of Laboratory medicine in diagnosis of Acute MI has increased tremendously.

Introduction of high sensitive and specific cardiac troponin assays in the latter part of the $20^{\text {th }}$ century, along with enzymes to detect myocardial damage, has given a pivotal role to the laboratory in the diagnosis and the follow up of cardiac cases.

Cardiac troponins ( $T$ and $I$ ) have been found to be superior due to their higher sensitivity to minor myocardial injury and its high specificity to cardiac damage. Troponin I appear as early as 6 hours after onset of MI, when the CK-MB fraction of Creatine Kinase enzyme also becomes detectable. Peak levels of troponin are attained by 24 hours and remain elevated

Article received on 10 OCT 2016, Published on 31 OCT 2016,

Rachel Jacob ${ }^{I}$,

${ }^{I}$ Additional Professor, Dept of Biochemistry,

Nizam's Institute of Medical Sciences,

Punjagutta, Hyderabad-500082

Corresponding Author: Rachel Jacob,

Email: rachel.jacob@gmail.com for several days. Troponin I may be useful for cases which are brought in delayed for admission, but the prolonged release pattern makes it difficult to be used serially for diagnosis of re-infarction, where CK-MB continues to play an important role.

Also elevation of serum troponin is related to the severity of the infarct, so that a microscopic infarct may lead to a troponin elevation for a few hours only. Hence caution must be exercised in the use of troponin testing. In the absence of ischemic changes, elevated troponin levels should prompt a search for the cause of other nonischemic conditions which can cause myocardial necrosis.

The cardiac enzymes used in the diagnosis of AMI have been Creatine Kinase and its iso-enzymes, Aspartate Transaminase and Lactate dehydrogenase and its iso-enzymes. AST and LDH rise much later i.e 24-72 hours after onset of symptoms, are nonspecific and hence not very useful.

Total CK levels are nonspecific but the CK-MB iso-enzyme (CK-2) elevation of $4 \%$ or more indicates MI. CK-MB rises within 4-6 hours after the onset of symptoms, peak levels are seen within 24 hours and persists for 2-3 days. This makes it an ideal marker to monitor re-infarcts and most importantly to mark successful thrombolytic therapy, since the circulating levels are elevated on reperfusion therapy. Creatine Kinase $-\mathrm{MB}(\mathrm{CK}-2)$ exists in 2 isoforms, CK-MB21 plasma form and CK-MB 22, tissue form, which are in equilibrium in normal state. The CK-MB 22 is elevated prior to CK-MB 21 in AMI ( $>2.5 \mathrm{IU} / \mathrm{L}$ is diagnostic) and the ratio of the 2 isoforms:

CK-MB 22/ CK-MB 21, which when exceeds $1.7 \%$, is suggestive of MI. It has been shown to have a sensitivity of $92 \%$ and a specificity of $95 \%$ for early detection of AMI, within 6 hours of infarct. Also this ratio is the best discriminant for successful reperfusion. But the use of 
these isoforms has been limited and does not obviate the need to assess the elevations in CK-MB.

CK-MB mass, which indicates the total active and inactive enzyme, appears an hour earlier than CK-MB, is more sensitive and the \% Relative Index: which is calculated as the ratio of CK-MB mass / CK Total X 100; when it is $>5 \%$ is an indicator of AMI, especially in the Non-ST elevation MI, where CK-MB elevation is small.

So the ideal marker should be one which is sensitive, specific, reliable and easy to assay with quick turnaround time and of prognostic use. In the present scenario a "must have" in every emergency setup is the troponin I test and the CK-MB mass which is the most useful for early detection and CK-MB activity for continuous monitoring in patients undergoing reperfusion therapy and for early detection of reinfarction.

\section{REFERENCES}

1. Nomenclature and Criteria for Diagnosis of Ischemic Heart Disease. Report of the Joint International Society and Federation of Cardiology/World Health Organization Task Force on Standardization of Clinical Nomenclature. Circulation 1979;59:607-8.

2. Alpert J, Thygesen $\mathrm{K}$ for the Joint European Society of Cardiology/American College of Cardiology Committee. Myocardial infarction redefined - a consensus document of the Joint European Society of Cardiology/American College of Cardiology Committee for the Redefinition of Myocardial Infarction. Eur Heart J 2000;21:1502-13.

3. HammCW. Acute coronary syndromes. The diagnostic role of troponins. Thromb Res. 2001;103:S63S69.

4. Apple FS, Wu AH, Jaffe AS. European Society of Cardiology and American College of Cardiology guidelines for redefinition of myocardial infarction: how to use existing assays clinically and for clinical trials. Am. Heart J.2002;144:981-986.

5. Panteghini M, Pagani F, Bonetti G. The sensitivity of cardiac markers: an evidence-based approach. Clin. Chem. Lab. Med. 1999;37:1097-1106.
6. Mueller C; Use of high-sensitivity troponin for the diagnosis of acute myocardial infarction. Coronary Artery Dis. 2013 Dec;24(8):710-2.

7. Chan D, Ng LL; Biomarkers in acute myocardial infarction. BMC Med. 2010 Jun 7;8:34. 